\title{
Computational Imaging of Light in Flight
}

\author{
Matthias B. Hullin \\ Institute of Computer Science II, University of Bonn, Regina-Pacis-Weg 3, 53113 Bonn, Germany
}

\begin{abstract}
This is an author-prepared version (Sections 3 and 4 revised and extended). Copyright Notice:
Matthias B. Hullin, "Computational Imaging of Light in Flight," Optoelectronic Imaging and Multimedia Technology III, Qionghai Dai, Tsutomu Shimura, Editors, Proc. SPIE 9273, 9273-40 (2014).

Copyright 2014 Society of Photo-Optical Instrumentation Engineers. One print or electronic copy may be made for personal use only. Systematic reproduction and distribution, duplication of any material in this paper for a fee or for commercial purposes, or modification of the content of the paper are prohibited.
\end{abstract}

DOI: http://dx.doi.org/nn.nnnn/nn.nnnnnn (definitive SPIE version yet to appear)

\begin{abstract}
Many computer vision tasks are hindered by image formation itself, a process that is governed by the so-called plenoptic integral. By averaging light falling into the lens over space, angle, wavelength and time, a great deal of information is irreversibly lost. The emerging idea of transient imaging operates on a time resolution fast enough to resolve non-stationary light distributions in real-world scenes. It enables the discrimination of light contributions by the optical path length from light source to receiver, a dimension unavailable in mainstream imaging to date. Until recently, such measurements used to require high-end optical equipment and could only be acquired under extremely restricted lab conditions. To address this challenge, we introduced a family of computational imaging techniques operating on standard time-of-flight image sensors, for the first time allowing the user to "film" light in flight in an affordable, practical and portable way. Just as impulse responses have proven a valuable tool in almost every branch of science and engineering, we expect light-in-flight analysis to impact a wide variety of applications in computer vision and beyond.
\end{abstract}

Keywords: Transient imaging, AMCW time-of-flight sensing, multi-path inversion

\section{INTRODUCTION}

A major challenge-if not the challenge-in computer vision stems from the fact that visual data is highly ambiguous: given any two-dimensional image, there are infinitely many possible hypotheses of real-world scenes that would explain what we see. In large part, this ambiguity can be traced back to the very mechanism of image capture: every pixel value of our camera is an integral of the so-called plenoptic function ${ }^{1}$ over space (the extent of a pixel location), angle (the aperture of the camera), wavelength (the spectral response) and time (the shutter period). Light contributions that reached the same sensor location on different paths or at different times are thus mixed irreversibly. As an immediate consequence, many common problems such as deblurring, motion tracking as well as the estimation of geometry, material reflectance and illumination are still considered unsolved even after decades of active investigation. It is only in the last few years that we have seen the development of novel imaging modalities that sample the temporal, angular and spectral dimensions to produce high- dimensional plenoptic images, facilitating some of those hard tasks.

Along similar lines, the highly promising research direction of transient imaging has emerged that introduces time resolution fast enough to resolve non-stationary light distributions in real-world scenes (see Fig. 1 for a synthetic example). Just like the capture and characterization of impulse responses has proven a valuable tool in almost every branch of science and engineering, the advent of transient imaging is expected to have a significant impact on a wide variety of applications in computer vision and beyond. ${ }^{2-4}$ Yet, such measurements have so far been expensive and hence hard to obtain. With our recent development of a computational imaging scheme using affordable and widespread AMCW time-of-flight imagers, ${ }^{5-7}$ we have lowered the entry barrier for this promising imaging modality, making it significantly more practical and bringing it within financial reach of most imaging labs.

E-mail: hullin@cs.uni-bonn.de. The work presented in this invited talk was done in close collaboration with Felix Heide, James Gregson, Lei Xiao, Wolfgang Heidrich (all of the University of British Columbia, Vancouver, Canada), as well as Hendrik Lensch (University of Tübingen, Germany) and Johannes Hanika (Karlsruhe Institute of Technology, Germany). 
The remainder of this paper is structured as follows. We will start by introducing the transient imaging problem and providing an overview of the state of the art in Section 2. Section 3 will review a computational technique for the reconstruction of transient images using data obtained from affordable AMCW time-of-flight imagers. In Section 4, we will show an example transient image and demonstrate use of transient data in secondary inverse problems using the example of non-line-of-sight imaging.
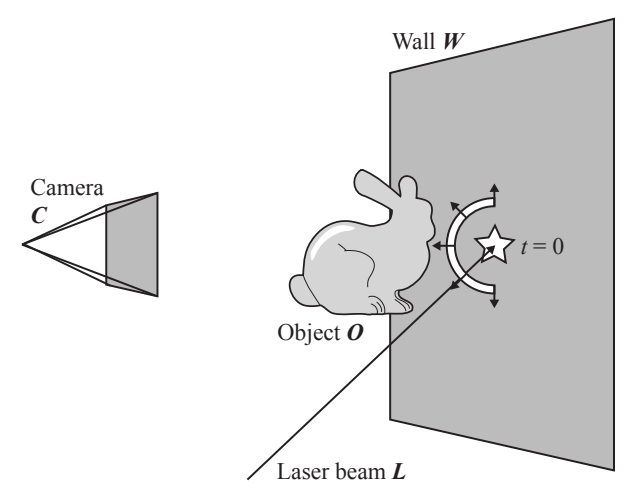
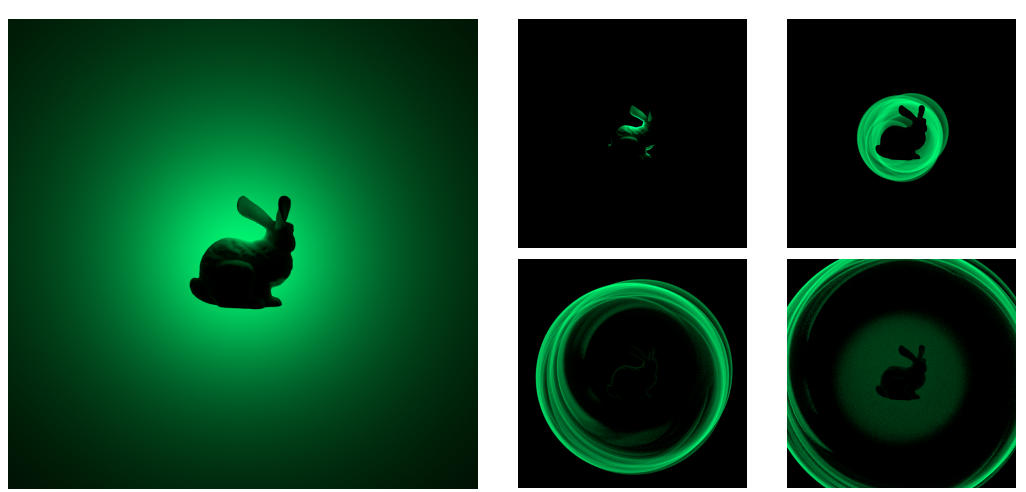

Figure 1. Transient simulation of indirect and diffuse reflection of a short laser pulse from a wall to the "Stanford Bunny" model and back to the wall. Left: sketch of the simulated scene. Center: steady-state solution (not time-resolved). Right: four snapshots of the transient image at different times. Note how much structure the time discrimination adds to the reflection on the wall (outgoing ring structures). All of this light was reflected off the back side of the bunny and hence a valuable source of information about a part of the object that is outside the direct line of sight of both camera and light source. This global illumination renderer was developed by Johannes Hanika in 2010 and took about 20 minutes to produce these results on a then-up-to-date PC with 16 CPU cores.

\section{PROBLEM DEFINITION AND RELATED WORK}

\subsection{THE TRANSIENT IMAGE}

The transient image $I_{A, B}(x, y, \tau)$ contains, in signal processing terms, the spatio-temporal response of a scene to illumination by an ultrashort pulse of light emitted from point $A$ and observed by a camera in a different point $B$. The scale of phenomena and effects described by a transient image $I$ defines the temporal resolution at which it must be sampled. The connection between distance and time is given by the speed of light $\left(c \approx 3 \times 10^{8} \mathrm{~m} / \mathrm{s}\right.$ in vacuum), e.g., within 1 nanosecond light would traverse roughly 1 foot of free space.

\subsection{PRIOR WORK IN ULTRAFAST IMAGING}

The measurement of an optical impulse response implies the use of a controlled light source and a camera, both temporally variable, and a system bandwidth high enough to resolve the time scale on which the phenomena of interest are happening. Due to the short time scales involved in light propagation (picoseconds to nanoseconds), this is a challenging problem. A variety of high-speed photography techniques have been developed that are potential candidates for transient imaging in indoor settings. The overview provided here is not exhaustive; instead, we recommend interested reader to use the our references as a starting point for literature exploration.

Traditional photography with fast shutter designs. The use of traditional camera designs with mechanical shutters can be ruled out for centimeter-scale transient imaging, no matter how sophisticated the mechanism. Although some designs employing rotating prisms and mirrors can yield temporal resolutions of less than $1 \mathrm{~ns}$-at least theoretically,$-{ }^{8}$ their practical challenges are manifold and include not only structural issues but also less obvious ones such as the need for vacuum conditions. ${ }^{9}$ Today's state of the art in temporal resolution is held by gated image intensifiers that can reach nominal shutter times down to $200 \mathrm{ps}$, still slightly longer than required for centimeter-scale transient imaging. However, they require extremely fast switching of high operating voltages, high optical peak powers, and they do not generalize well to repetitive shooting and a dense sampling on the temporal domain. 
Fast opto-electronics. Individual certain opto-electronic detectors (photodiodes) do achieve sub-nanosecond rise times, but the processing of such signals requires nontrivial instrumentation effort with multi-GHz analog bandwidth, ${ }^{10}$ and scaling such solutions to full-frame capture (thousands to millions of pixels) is not an option. The frame rate at which traditional (CCD and CMOS-type) imagers can be operated is constrained by principal limitations, such as the loss of light due to short integration times, and technological challenges, such as the need for sufficiently fast read-out mechanisms. ${ }^{11}$ Even the most advanced solutions on the market offer no more than a few thousand frames per second and thus fall short of our requirements for transient imaging by several orders of magnitude. It remains to be seen what recent pixel designs such as CMOS-based single-photon avalanche diode arrays ${ }^{12}$ will be able to contribute to transient imaging.

Optical gating, holography, and streak cameras. The development of pulsed lasers in conjunction with non-linear optical media has given rise to a number of techniques to characterize short optical events. Seeking to capture transient images even with slow detectors, a mature direction of research seeks to switch light with light, constructing gates that only transmit light for a short periods of time. ${ }^{13-15}$ The major drawback of these approaches is that they sample light only along a single ray, and hence require additional effort for mechanical scanning. This limitation is in part overcome by holographic techniques ${ }^{16,17}$ and streak tube cameras that smear out a single scanline over time. ${ }^{18}$ As far as transient imaging is concerned, both the holographic and the streak camera approach are champions in terms of spatial and temporal resolution. However, they require extremely careful set-up, very high optical powers, hour-long capture times and access to a laser laboratory, and so are not within reach for the casual user.

Candidate technology: AMCW time-of-flight sensors. Over the recent decade, we have witnessed the emergence of time-of-flight sensors that deliver precise per-pixel range measurements. The most common implementation, the amplitude-modulated continuous-wave (AMCW) technique, features a lock-in amplifier per pixel. Devices implementing this approach are also referred to as photonic mixer devices ${ }^{19}$ or demodulation pixels. ${ }^{20}$ Each pixel of such CMOS-based sensors can direct the charge from incoming photons to two or more storage sites, ${ }^{19,21}$ an idea that has recently been extended to multi-bucket sensors. ${ }^{22}$ In their typical usage scenario, AMCW imagers are modulated with a periodic function $f_{\omega}(t)$ and used in combination with a continuous-wave (CW) light source that is modulated at the same frequency with function $g_{\omega}(t)$ as illustrated in Fig. 2. Assuming that the light has experienced delay $\tau$ and attenuation $a$ on its way to the sensor, resulting in a modified illumination function $\tilde{g}_{\omega}(t)=a g_{\omega}(t-\tau)$, the resulting correlation measurements are given as

$$
\begin{aligned}
h_{\omega} & =\int_{0}^{T} f_{\omega}(t) \tilde{g}_{\omega}(t) d t \\
& =a \int_{0}^{T} f_{\omega}(t) g_{\omega}(t-\tau) d t
\end{aligned}
$$

where $T$ is the integration time (which, for the sake of simplicity, is assumed to be an integer multiple of the modulation period) and $\omega$ is the modulation frequency. In traditional range imaging, the task is to fit the time of flight $\tau$ and the amplitude $a$, which can be achieved with high repeatability using four such measurements taken at different relative phase shifts (in steps of $90^{\circ}$ ).

\section{RECONSTRUCTING TRANSIENT IMAGES FROM AMCW MEASUREMENTS}

To overcome of the practical limitations of existing transient imaging hardware, we propose to use lock-in sensors in conjunction with a computational reconstruction step to acquire transient images. In recent work, we demonstrated that data captured using this technology encodes transient images in a linear way. ${ }^{5}$ The foundation of our work is an image formation model that extends the common assumption that light only reaches the sensor on a single path (corresponding to direct reflections by the scene). To this end, we generalize $\tilde{g}_{\omega}(t)$ into an integral expression:

$$
\tilde{g}_{\omega}(t)=\int_{\tau_{\min }}^{\tau_{\max }} a(\tau) g_{\omega}(t-\tau) d \tau,
$$




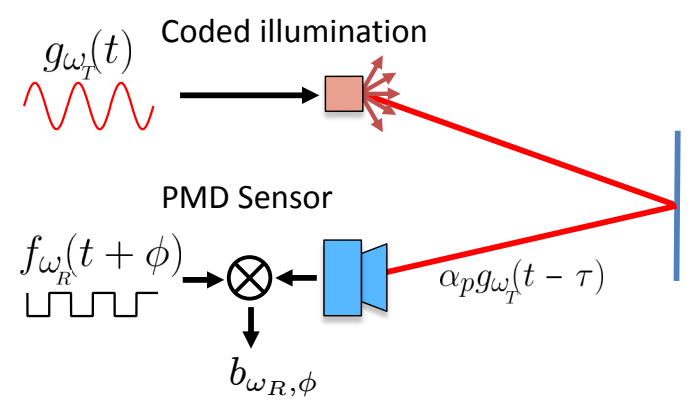

Single path ToF

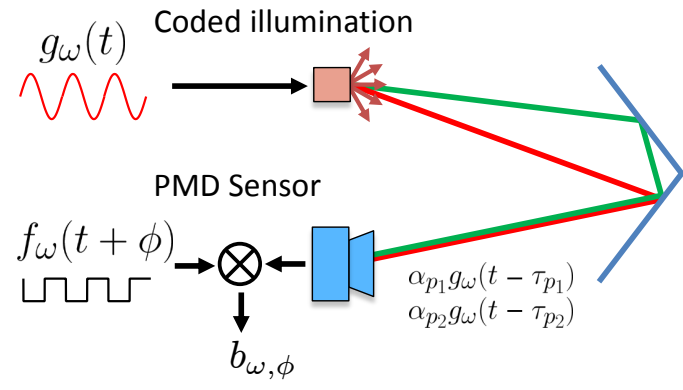

Multi path ToF

Figure 2. Time-of-flight depth imaging assuming direct reflection (left; single light path contributing to pixel) and global illumination (right; multi-path scattering). Illustrations courtesy of Felix Heide.

where $a(\tau)$, in turn, is the transient response of a pixel. It can be interpreted as a path-space integral that returns the combined intensity of all contributing light paths of travel time $\tau$ :

$$
a(\tau)=\int_{\mu(p)=\tau} a(p) d p
$$

We use Eq. 3 to turn Eq. 1 into a full global illumination model for the measured value resulting from the multitude of ways in which the light can scatter back into the camera:

$$
h_{\omega}=\int_{0}^{T} f_{\omega}(t) \int_{\tau_{\min }}^{\tau_{\max }} a(\tau) g_{\omega}(t-\tau) d \tau d t
$$

In order to reconstruct $a(\tau)$, we discretize $\tau$ into a finite number of bins, turning the function $a$ into a vector $\mathbf{i}$ of sampled values and the inner integral into a sum:

$$
\begin{aligned}
i_{k} & =\int_{\tau_{k}}^{\tau_{k+1}} a(\tau) d \tau \\
h_{\omega} & \approx \sum_{k} i_{k} \underbrace{\int_{0}^{T} f_{\omega}(t) g_{\omega}\left(t-\tau_{k}\right) d t}_{=c_{\omega, \tau}},
\end{aligned}
$$

where the correlation coefficient $c_{\omega, \text { tau }}$ is no longer signal dependent and hence can be calibrated for the light sourcecamera setup. We note that the measured outcome is linear in i. By varying the modulation frequency $\omega$, we obtain a vector of measurements $\mathbf{h}$ that is related to the discretized transient image $\mathbf{i}$ through a matrix $\mathbf{C}$ containing the correlation coefficients, which is our linear model of image formation:

$$
\mathbf{h}=\mathbf{C i}
$$

Thus, the recovery of a transient image from lock-in measurements is a linear inverse problem, albeit a difficult one to solve. Due to a maximum modulation frequency in the order of $100 \mathrm{MHz}$ (corresponding to 3 meters of light propagation), the linear system is ill-conditioned, ruling out a naïve least-squares solution and requiring effective regularization strategies. A common device for the regularization of closely related inverse problems like deconvolution or tomographic reconstruction are sparsity-inducing $\ell_{1}$ gradient priors, for instance total variation (TV). Since the transient image underlies physical constraints of light transport in a scene, it is reasonable to also consider physically based models with a small number of parameters. ${ }^{23}$ The use of such models for regularization turns the problem non-convex and calls for specialized solvers, unless the number of parameters is small enough to use a basis pursuit approach. In recent work, we introduced the use of exponentially modified Gaussian distributions in a sparse convolutional framework to reconstruct temporally dense pixel responses. $^{2}$ 


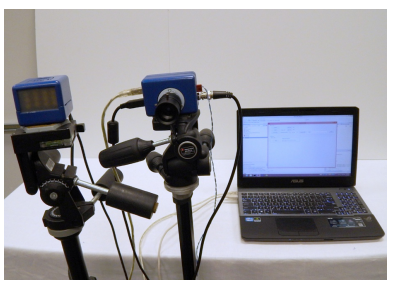

(a)

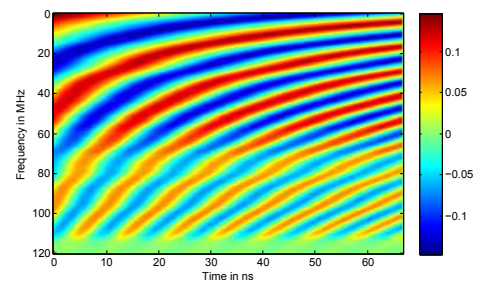

(b)

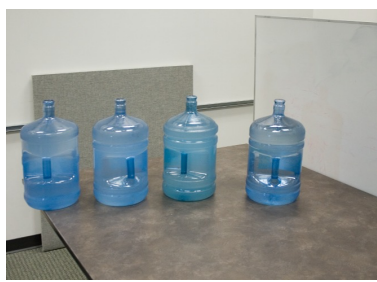

(c)

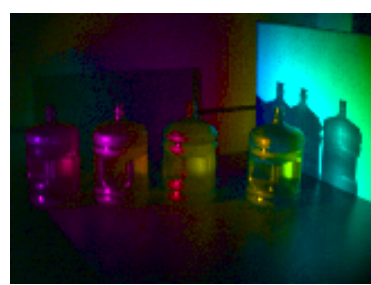

(d)

Figure 3. From left to right: (a) Photo of our imaging setup. (b) Measured correlation matrix. (c) Photo of a scene composed of several bottles filled with scattering water. The transient light source is located to the left, just outside the field of view. (d) Color-coded rendering of a transient image of the same scene. Hue encodes the time of flight for the main intensity peak of the reconstruction.
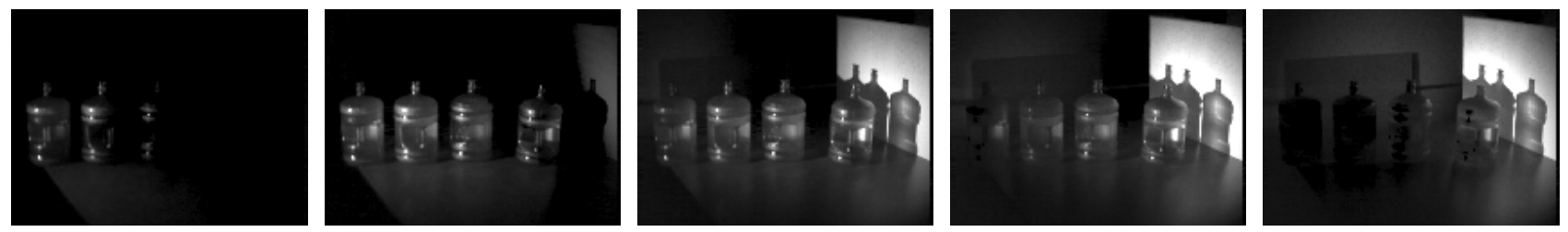

Figure 4. Time slices from a transient image captured with our setup, and reconstructed using a spatio-temporal sparsity prior. ${ }^{5}$ As light propagates through a scene containing several bottles filled with water, we see first reflection of the surface of the bottles, followed by scattering inside the bottles, caustics, and light scattering onto the back wall.

\section{INTERPRETATION OF TRANSIENT IMAGES}

Fig. 3 shows a scene with several bottles, filled with water and a small amount of milk to create scattering. Slices of the corresponding transient image as reconstructed using the method described in our 2013 SIGGRAPH paper ${ }^{5}$ are shown in Fig. 4. In the first frame, the wavefront has just reached the front of the leftmost bottles, and is reflecting off their surface. In the second frame, scattering effects are becoming visible in the bottles. Next, the light reaches the far wall, showing caustics of the light transport through the bottles. Indirect illumination of the back wall from light scattered in the bottles appears in the fourth frame. This light continues to illuminate the back ball even after the bottles themselves have darkened (last frame).

\subsection{MULTI-PATH ANALYSIS}

Not only does the observation of light in flight offer a unique perspective on time and space, it can also be used to reason about scene properties. Virtually all fields of remote sensing that employ time-of-flight measurements are affected by the problem of multi-path scattering, i.e. signals that are reflected on other paths than the one intended. Traditionally, in lack of better options, multi-path reflections have mostly been treated as a pollutant. ${ }^{24}$

Only recently, significant success has been reported in exploiting multi-path scattering as an additional source of information in the geosciences ${ }^{25,26}$ or radar localization. ${ }^{27}$

Underlying all multi-path analysis is the basic assumption that the transient image $\mathbf{i}$ results from light propagating through a scene that is defined by a set of parameters $\mathbf{v}$. The image formation model describes this light transport process and is expressed as a function $\mathbf{p}()$ :

$$
\mathbf{i}=\mathbf{p}(\mathbf{v})
$$

Generative models / analysis-by-synthesis. In order to solve the inverse problem of recovering $\mathbf{v}$ given $\mathbf{i}$, several paths can be taken. If light transport is very complex but well-understood, and only a small number of parameters is to be recovered, then an analysis-by-synthesis method is the most general approach, although very costly to compute. As of today, there are no general global illumination renderers capable of producing transient images and fast enough to be suited for use inside an optimization loop. In more constrained situations, for instance, we were able to demonstrate a GPU-based approach based on the radiosity method that closely approximates the transient image shown in Fig. 1 and executes within a small fraction of a second - the results can be seen in Fig. 5. The inverse problem can then be formulated 

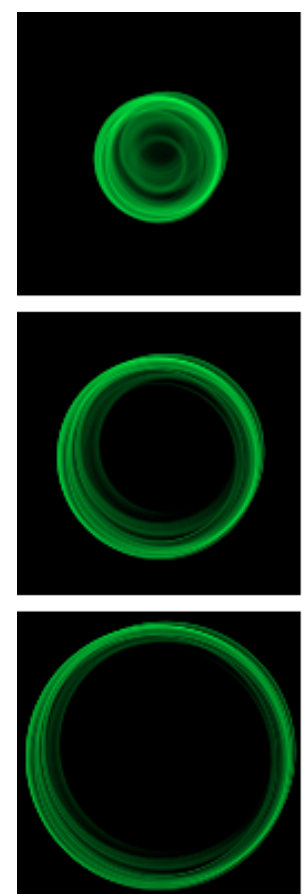

Raw
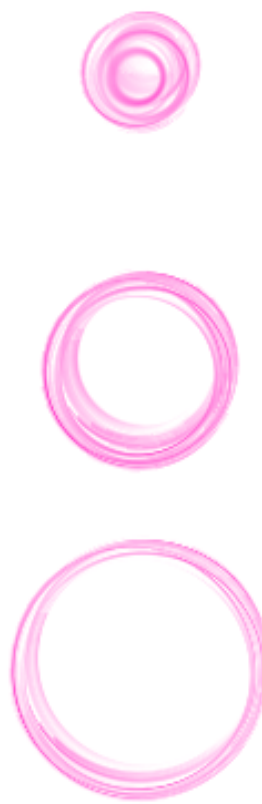

Shadowing
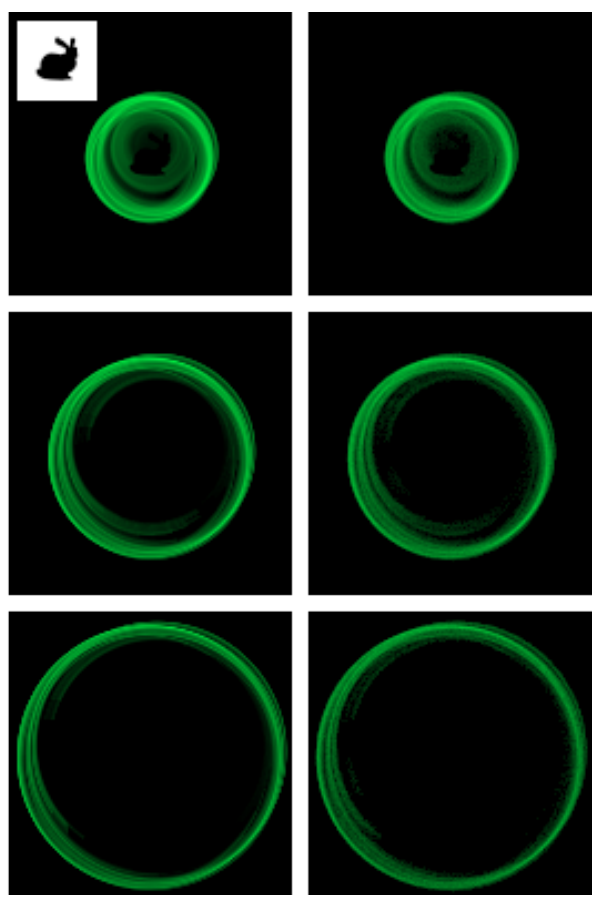

Result
Reference

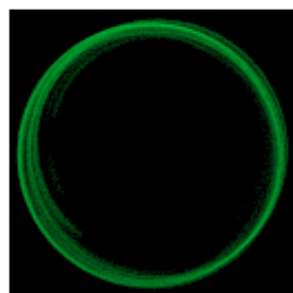

Figure 5. Approximation of transient light transport using single-bounce temporal radiosity, shown at different moments in time (rows). From left to right: Raw temporal radiosity term, self-shadowing correction term, net temporal radiosity including silhouette mask (inset), pathtraced reference with 100,000 samples per pixel. Rendering times are 0.12 seconds for the temporal radiosity solution (GPU) vs. 21.3 minutes for the pathtracer (on 16 CPU cores).

as the following optimization problem. We note, however, that the problem is, in general, not convex and a global solution therefore not guaranteed:

$$
\mathbf{v}_{\mathrm{opt}}=\underset{\mathbf{v}}{\arg \min } \frac{1}{2}\|\mathbf{i}-\mathbf{p}(\mathbf{v})\|_{2}^{2}+\Gamma(\mathbf{v})
$$

where $\Gamma(\mathbf{v})$ is a problem-specific regularizer. Fig. 6 shows the reconstruction of an approximate geometry from a simulated transient image. The object geometry was inferred using an unregularized gradient descent wrapped around the radiositybased implementation of the light transport operator. The object is represented as a tessellated spherical blob, where the vector $\mathbf{v}$ contains all the vertex positions. The optimization loop displaces and deforms the mesh in an effort to minimize error function Eq. 12, eventually converging to a shape that displays distinct features of the actual unknown side. Although the use of such technique for the reconstruction of real-world, highly detailed 3D object geometry has yet to be demonstrated, we expect the approach to fill in where a linearization of the image formation model (as explained in the following) is not possible. The generality comes at a significant cost, though: the result took over 3 days to compute on a recent graphics processor, and required several million evaluations of the transient image given the current geometry hypothesis - each evaluation amounting to rendering a full transient video using a simplistic global illumination solver.

Linearized models The inverse problem of solving Eq. 9 for the parameter set $\mathbf{v}$ is a challenge of its own, but things become even harder when $\mathbf{i}$ is not accessible directly but only in coded form. This is exactly the case when using AMCW time-of-flight setup, where the relation between the latent transient image $\mathbf{i}$ and the measurements $\mathbf{h}$ is given by Eq. 8 . In Heide et al. $2014,{ }^{6}$ we dealt with this situation by deriving a linear light transport operator $\mathbf{P}$ that acts on a volume $\mathbf{v}$ approximately describes how much is scattered from each volume element into each camera pixel. Such linearized models cannot deal with phenomena such as non-local scattering, shadowing or occlusion, but they allow for a joint inversion of the light transport and the image formation models. The joint forward model is given as

$$
\mathbf{h}=\mathbf{C P} \mathbf{v}
$$



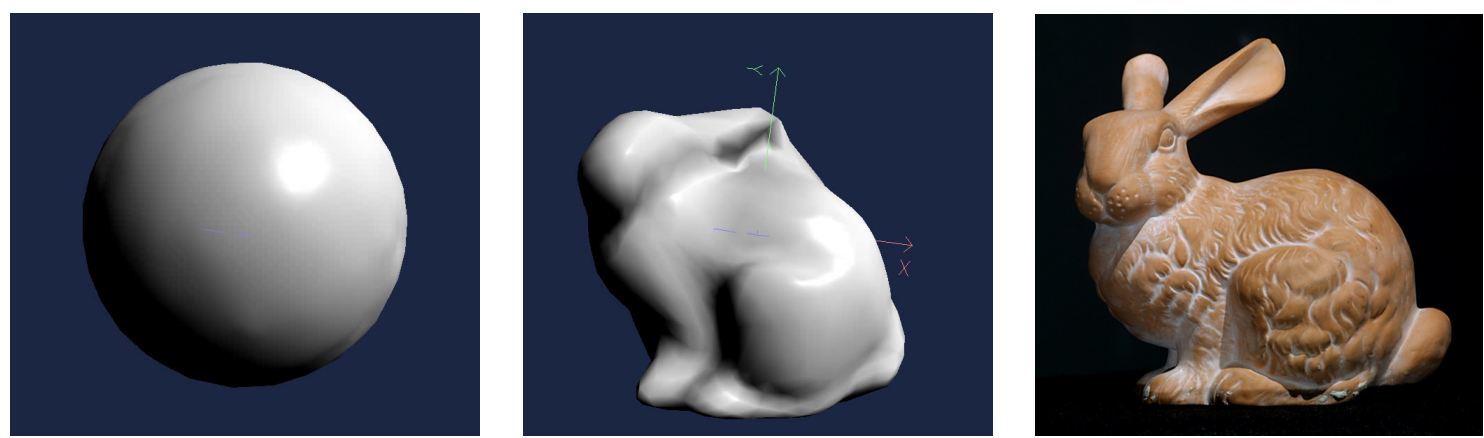

Figure 6. Demonstration of the analysis-by-synthesis approach on a simulated example. The "measurement", i.e., the left-hand side of Eq. 12 is taken from Fig. 1 (simulated transient image). Left: $\mathbf{v}$ is initialized as a spherical blob. Center: The optimized $\mathbf{v}$ has converged to a distinctly bunny-like shape. Right: Photo of the actual Stanford Bunny (courtesy of Marc Levoy).

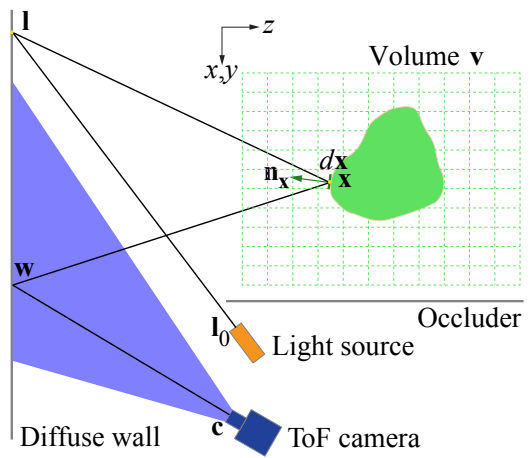

(a) Top view

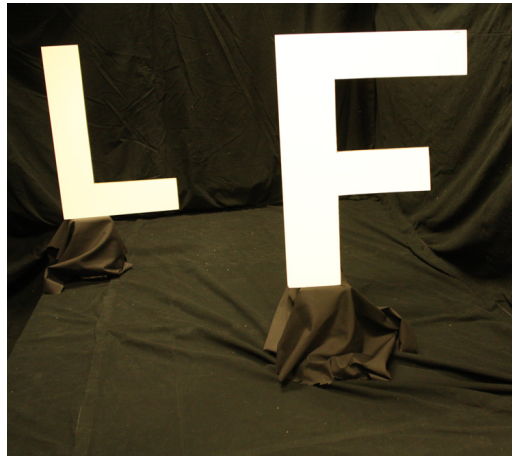

(b) Unknown scene

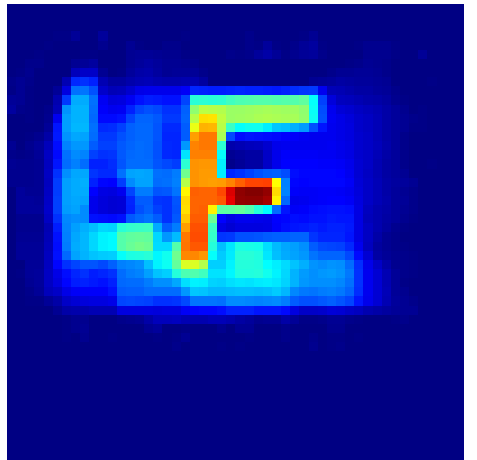

(c) Reconstructed depth map (red=near, blue $=$ far $)$

Figure 7. Looking around the corner on a shoestring budget. ${ }^{6}$ (a) Top view of our measurement scenario (to scale). A diffuse wall is illuminated by a modulated laser beam (red) and observed by a time-of-flight camera (blue). Even after two diffuse bounces off the wall, inversion of a linearized light transport model allows us to infer the geometry and albedo of objects within a bounding volume (green) that is completely occluded to both light source and camera, but visible from most locations on the wall. In this example, the shape of two letters cut from cardboard (b) becomes clearly visible in the reconstruction (c).

with the corresponding least-squares formulation of the inverse problem,

$$
\mathbf{v}_{\text {opt }}=\underset{\mathbf{v}}{\arg \min } \frac{1}{2}\|\mathbf{h}-\mathbf{C P v}\|_{2}^{2}+\Gamma(\mathbf{v})
$$

Since the transient image is only contained implicitly, any nonlinear prior that used to be required for its regularization can be skipped completely. It is replaced by an application-dependent regularizer applied directly in the reconstruction domain, i.e., on the volume v. In order to, out of all volumes that solve Eq. 11, selects the one that is the sparsest, i.e., the closest to describing the surface of an opaque object, we developed a set of sparsity-inducing penalties and derived the necessary numerical framework to solve for them. As a result, we were able to demonstrate the reconstruction of object albedo and geometry purely from $3^{\text {rd }}$-order diffusely reflected light off a wall (Fig. 7).

\section{CONCLUSION}

Using widely available and affordable AMCW range imagers, the reconstruction of transient images can be cast as an linear inverse problem. Transient data obtained from such imaging setups can not only serve to illustrate macroscopic light transport phenomena, but also be used to infer additional information about the albedo and geometry of objects that are outside the line of sight.

\section{ACKNOWLEDGMENTS}

Matthias Hullin is supported under the X-Rite Chair for Digital Material Appearance. 


\section{REFERENCES}

[1] Adelson, E. H. and Bergen, J. R., "The plenoptic function and the elements of early vision," in [Computational Models of Visual Processing ], 3-20, MIT Press (1991).

[2] Heide, F., Hullin, M. B., Xiao, L., Kolb, A., and Heidrich, W., "Imaging in scattering media using correlation image sensors and sparse convolutional coding," Opt. Express (in submission).

[3] O’Toole, M., Heide, F., Xiao, L., Hullin, M. B., Heidrich, W., and Kutulakos, K. N., "Temporal frequency probing for 5D transient analysis of global light transport," ACM Trans. Graph. (Proc. SIGGRAPH 2014) 33(4), 87:1-87:11 (2014).

[4] Velten, A., Willwacher, T., Gupta, O., Veeraraghavan, A., Bawendi, M., and Raskar, R., "Recovering threedimensional shape around a corner using ultrafast time-of-flight imaging," Nature Communications 3, 745 (2012).

[5] Heide, F., Hullin, M. B., Gregson, J., and Heidrich, W., "Low-budget transient imaging using photonic mixer devices," ACM Trans. Graph. (Proc. SIGGRAPH 2013) 32(4), 45:1-45:10 (2013).

[6] Heide, F., Xiao, L., Heidrich, W., and Hullin, M. B., "Diffuse mirrors: 3D reconstruction from diffuse indirect illumination using inexpensive time-of-flight sensors," IEEE Conf. on Computer Vision and Pattern Recognition (CVPR) (2014).

[7] Lin, J., Liu, Y., Hullin, M. B., and Dai, Q., "Fourier analysis on transient imaging by multifrequency time-of-flight camera," in [IEEE Conference on Computer Vision and Pattern Recognition (CVPR)], (June 2014).

[8] Igel, E., [Rotating Mirror Streak and Framing Cameras], SPIE Digital Library, SPIE Optical Engineering Press (1997).

[9] Li, J., Gong, X., and Wu, Q., “Camera bellows of rotating-mirror framing camera without principle[sic] error.” US Patent application US 20110002683 A1.

[10] Kirmani, A., Hutchison, T., Davis, J., and Raskar, R., "Looking around the corner using transient imaging," in [Proc. ICCV], 159-166 (2009).

[11] Goda, K., Tsia, K., and Jalali, B., "Serial time-encoded amplified imaging for real-time observation of fast dynamic phenomena," Nature 458(7242), 1145-1149 (2009).

[12] Burri, S., Maruyama, Y., Michalet, X., Regazzoni, F., Bruschini, C., and Charbon, E., "Architecture and applications of a high resolution gated spad image sensor," Opt. Express 22, 17573-17589 (Jul 2014).

[13] Giordmaine, J. A., Rentzepis, P. M., Shapiro, S. L., and Wecht, K. W., "Two-photon excitation of fluorescence by picosecond light pulses," Applied Physics Letters 11(7), 216-218 (1967).

[14] Duguay, M. A. and Mattick, A. T., "Ultrahigh speed photography of picosecond light pulses and echoes," Appl. Opt. 10, 2162-2170 (Sep 1971).

[15] Morimoto, A., Kobayashi, T., and Sueta, T., “A picosecond optical gate using photo-induced grating," Japanese Journal of Applied Physics 20, 1129-1133 (June 1981).

[16] Abramson, N., "Light-in-flight recording by holography," Opt. Lett. 3, 121-123 (Oct 1978).

[17] Kakue, T., Tosa, K., Yuasa, J., Tahara, T., Awatsuji, Y., Nishio, K., Ura, S., and Kubota, T., "Digital light-in-flight recording by holography by use of a femtosecond pulsed laser," Selected Topics in Quantum Electronics, IEEE Journal of 18(1), 479-485 (2012).

[18] Velten, A., Raskar, R., and Bawendi, M., "Picosecond camera for time-of-flight imaging," in [OSA Imaging Systems and Applications ], (2011).

[19] Schwarte, R., Xu, Z., Heinol, H., Olk, J., Klein, R., Buxbaum, B., Fischer, H., and Schulte, J., "New electro-optical mixing and correlating sensor: facilities and applications of the photonic mixer device," in [Proc. SPIE], 3100, 245253 (1997).

[20] Lange, R., Seitz, P., Biber, A., and Lauxtermann, S., "Demodulation pixels in CCD and CMOS technologies for time-of-flight ranging," Sensors and camera systems for scientific, industrial, and digital photography applications , 177-188 (2000).

[21] Gokturk, S., Yalcin, H., and Bamji, C., "A time-of-flight depth sensor - system description, issues and solutions," in [Computer Vision and Pattern Recognition Workshop, 2004. CVPRW'04. Conference on], 35 (2004).

[22] Wan, G., Li, X., Agranov, G., Levoy, M., and Horowitz, W., "CMOS image sensors with multi-bucket pixels for computational photography," IEEE J. Solid-State Circuits 47(4), 1031-1042 (2012).

[23] Wu, D., O’Toole, M., Velten, A., Agrawal, A., and Raskar, R., "Decomposing global light transport using time of flight imaging," in [Proc. CVPR], 366-373 (2012). 
[24] ATIS, "Alliance for telecommunication industry solutions. telecom glossary 2007, definition of multipath." http://www.atis.org/glossary/definition.aspx?id=3776 (2007).

[25] Gerstoft, P., "Inversion of seismoacoustic data using genetic algorithms and a posteriori probability distributions," The Journal of the Acoustical Society of America 95(2), 770-782 (1994).

[26] Nievinski, F. G. and Larson, K. M., "Forward modeling of gps multipath for near-surface reflectometry and positioning applications," GPS Solutions , 1-14 (2013).

[27] Setlur, P., Smith, G., Ahmad, F., and Amin, M., "Target localization with a single sensor via multipath exploitation," Aerospace and Electronic Systems, IEEE Transactions on 48(3), 1996-2014 (2012). 